\title{
Pelvic Angiography and the Diagnosis of Carcinoma of the Uterine Cervix
}

\author{
Mahmood Yoonessi, ${ }^{1}$ Stewart R. Reuter, ${ }^{2}$ Chien H. Meng ${ }^{2}$ and Seymour L. Romney ${ }^{3}$ \\ Departments of Obstetrics and Gynecology ${ }^{1}$ and Radiology, ${ }^{2}$ Albert Einstein College of Medicine, Bronx, New York, USA \\ ${ }^{3}$ University of Michigan Medical Center, Ann Arbor, Michigan, USA
}

\begin{abstract}
Yoonessi $M$, Reuter SR, Meng CH, Romney SL (Depts of Obstetrics and Gynecology and Radiology, Albert Einstein College of Medicine, Bronx, NY, and University of Michigan Medical Center, Ann Arbor, MI, USA). Pelvic angiography and the diagnosis of carcinoma of the uterine cervix.

Int J Gynaecol Obstet 17: 411-414, 1980

The hospital records and pelvic arteriograms of 31 patients with suspected, persistent or recurrent carcinoma of the uterine cervix were reviewed. The method used was found to be highly accurate in diagnosing and localizing tumors and was considered most helpful in suspected pelvic wall recurrences. Tumor encasement of the vessels can be mimicked by perivascular, postoperative and post radiation fibrosis. Tumor vessels and tumor stain are the better angiographic indicators of the presence of recurrent or persistent disease.
\end{abstract}

\section{INTRODUCTION}

Pelvic arteriography has been used in patients with carcinoma of the uterine cervix for diagnostic $(3-7,9)$ and therapeutic $(1,2,8)$ purposes. Its reliability and usefulness has reportedly been variable. This study was undertaken to further examine the value of pelvic angiography in patients with cervical carcinoma and to define its indications and limitations.

\section{MATERIALS AND METHODS}

The hospital records and pelvic angiograms of 26 patients seen at the Albert Einstein College of Medicine, Bronx, NY, and five patients seen at the University of Michigan Medical Center were reviewed. All patients had been previously treated for cervical cancer and were clinically suspected of having persistent or recurrent pelvic disease. Angiography was done with bilateral catheters placed selectively in the hypogastric arteries using a percuta- neous transfemoral approach. In some cases, a magnification technique was used. Angiographic diagnosis of the persistent or recurrent tumor was made on the basis of clear findings of one or more of the following: (1) a serrated or serpiginous type of encasement of pelvic arteries, (b) tumor vessels or tumor neovascularity, (c) tumor stain or blush or (d) arteriovenous shunting.

Findings at laparotomy (19 patients) or autopsy (4 patients) were compared and correlated with angiographic findings in 23 instances. Unexplored cases consisted of five patients with negative and three patients with positive pelvic arteriograms. Clinical, cytologic, and biopsy information was used to determine the accuracy rate of angiography in this group.

\section{RESULTS}

Angiographic diagnosis of the absence or presence and location of the tumor among laparotomy or autopsy patients was correct in $20(87 \%)$ and incorrect in 3 cases (13\%). When the diagnosis of recurrence was based on the presence of tumor vessels, with or without other findings, only one of 14 patients (7\%) had negative laparotomy and/or autopsy. One of three patients with presumed tumor encasement of the iliac vessels had pelvic fibrosis only. One patient with nondiagnostic hypervascularity was found to have recurrent tumor at the pelvic wall.

Five of the unexplored patients had negative pelvic arteriograms. These patients have been followed for at least two years following the initial clinical suspicion of persistent or recurrent cancer. They all remain well without evidence of disease and their intravenous urograms and cytology have remained negative. Of the three unexplored patients with pelvic angiograms positive for tumor two were confirmed by biopsy, and both patients died with cancer. The third patient with suspected tumor 


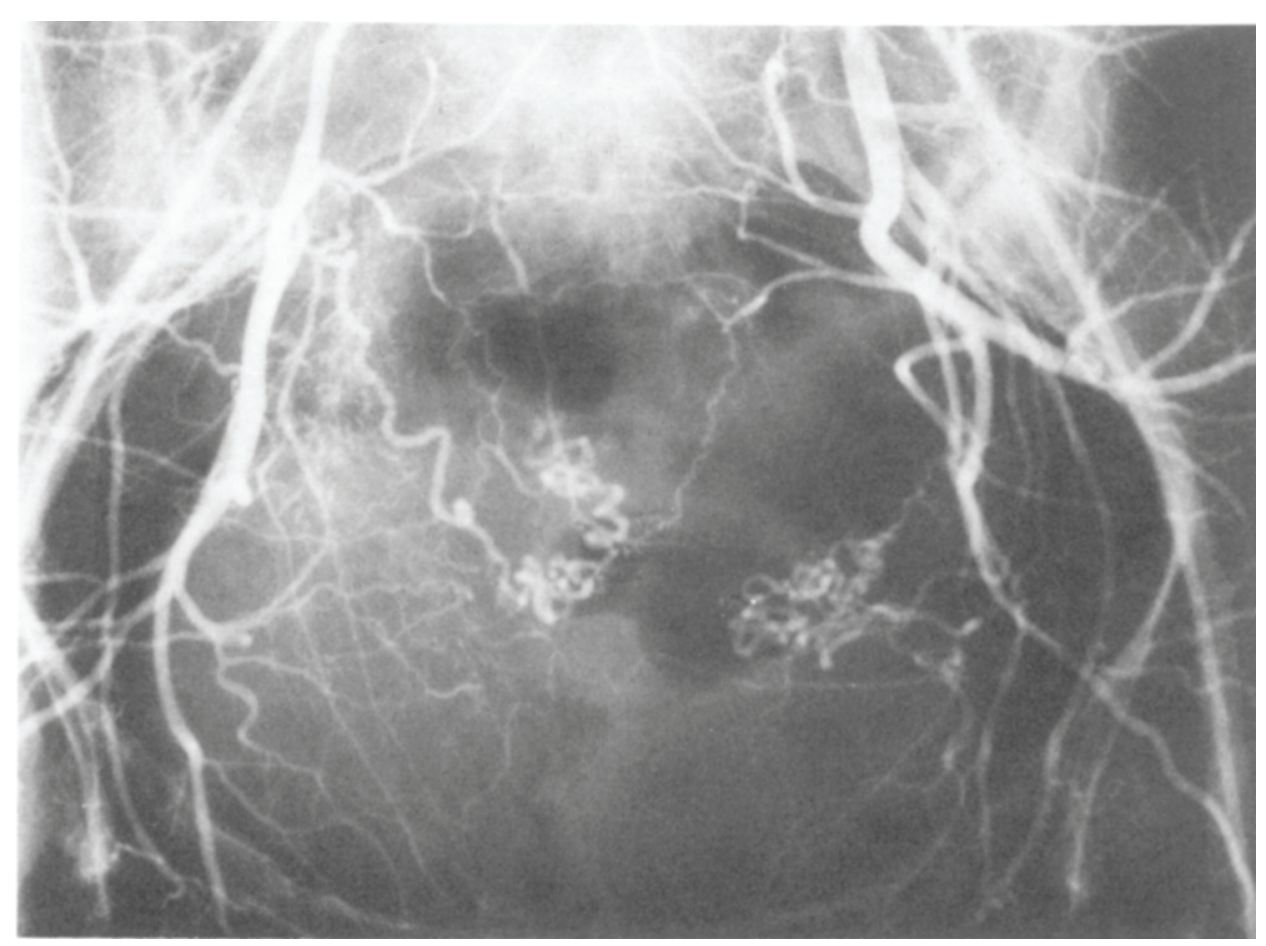

Fig. 1. Case 1: Simultaneous bilateral iliac angiograms in a woman with suspected recurrent cervical carcinoma. Many tumor vessels along the right pelvic wall between the internal iliac artery and the elevated uterine artery indicate recurrence. At laparotomy, recurrent squamous cell carcinoma was found along the right pelvic wall. The uterus was free of tumor.

encasement of the pelvic vessels has remained well for four years and has pelvic fibrosis but no clinical or cytopathologic evidence of tumor.

\section{DISGUSSION}

Conrady et al (3), in 1966, reported the results of pelvic arteriography with or without lymphangiography in 19 cases of cervical carcinoma and concluded that the study is most valuable in advanced and recurring cases, and as a means of defining the true extent of the disease. Lang and Greer (7) compared angiographic and surgical findings in $22 \mathrm{pa}-$ tients and noted that the accuracy rate of their clinical staging of this malignancy could improve significantly by taking arteriographic findings into consideration. Another smaller series has been reported (10) which suggested a variable reliability. With the current concepts of pretreatment surgical staging, more emphasis is placed upon laparotomy and biopsies to determine the actual extent of lymphatic and serosal dissemination of most cases of cervical carcinoma. This tends to limit the indications for pretherapy pelvic arteriography. The results of this study indicate that, in treated cases, angiography remains a valuable method of assessing and, in some instances, identifying patients with persistent or recurrent pelvic disease. The $87 \%$ accuracy rate of pelvic arteriography in determining the absence or presence and location of persistent and/or recurrent tumor attests to its usefulness. Admittedly, some patients with suspected persistent or recurrent cervical cancer can be evaluated easily by biopsy of a visible central lesion or a nodular parametrium and do not require angiographic confirmation. However, many suspected early pelvic wall recurrences are difficult to prove or disprove. Furthermore, when the presence of disease is suspected in patients with radiation complications (eg, fistulas with superimposed inflammatory reactions), required diagnostic surgical intervention can be a major undertaking. It is in these cases that pelvic angiography is of the greatest value. It helps to identify patients with persistent or recurrent cancer and defines the location and extent of their disease.

Pelvic fibrosis only was found in two of four patients in whom the angiographic interpretation was the presence of tumor on the basis of encasement of the internal iliac artery and its major branches. This would suggest that tumor encasement can be mimicked by perivascular postoperative changes and radiation fibrosis, and that tumor vessels and 


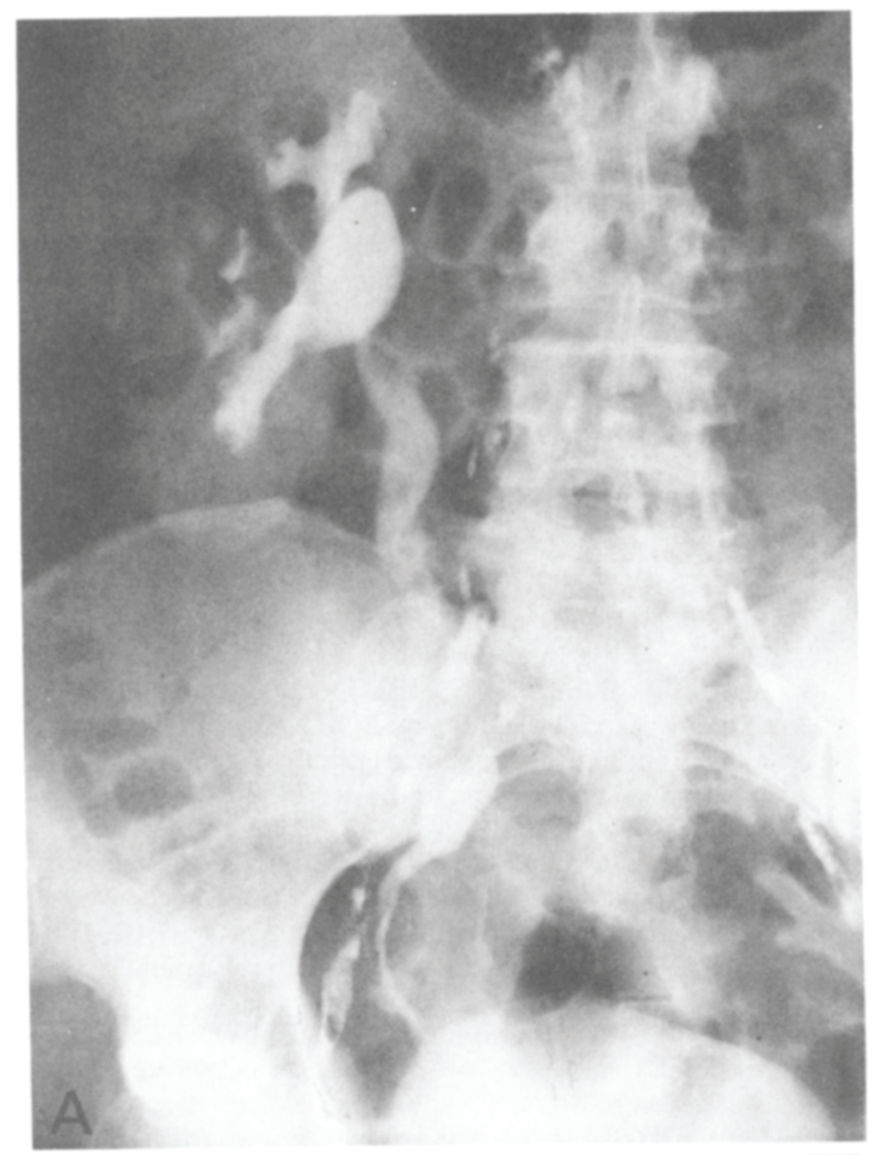

Fig. 2. Case 2: Intravenous urography and bilateral internal iliac angiography in a woman with suspected recurrent cervical carcinoma. A, Intravenous urogram reveals that the ureter and collecting structures on the right are dilated and shows evidence of partial obstruction of the ureter at the level of the pelvic brim. Pretreatment intravenous urogram was normal. B, Right internal iliac angiogram reveals no tumor vessels. The uterine artery has a normal position and caliber and supplies the usually tortuous branches through the myometrium. C, Left internal iliac angiogram reveals no tumor vessels or other abnormalities to suggest recurrent carcinoma. The angiographic findings were confirmed at laparotomy.
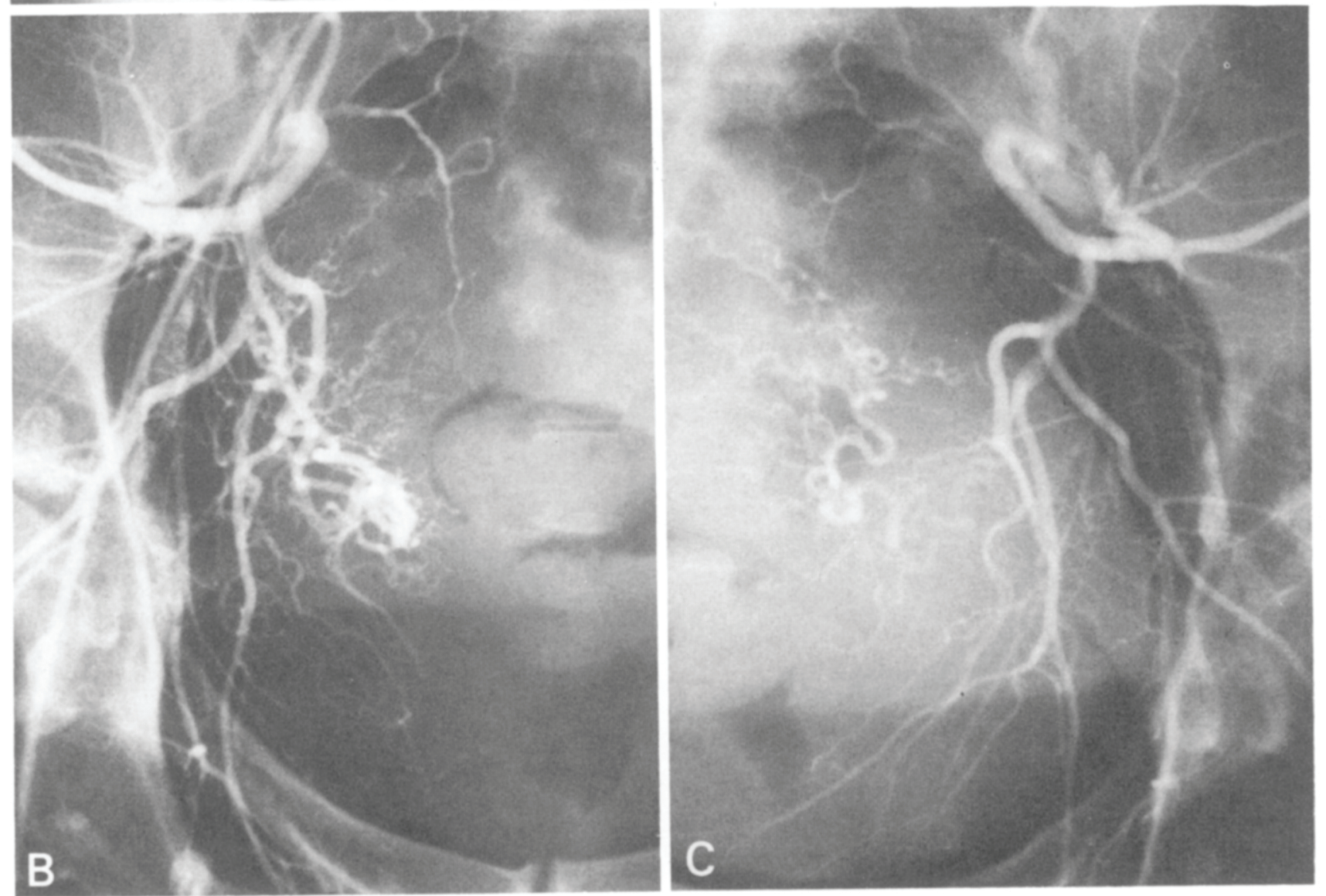
tumor stain are better indicators of the presence or absence of persistent or recurrent tumor.

Embolization of the hypogastric arteries $(1,2)$ and pelvic perfusion of chemotherapeutic agents (8) constitute other potential indications for pelvic arteriography in patients with cervical carcinoma.

The following case histories demonstrate the potential usefulness of angiography in the evaluation of patients with suspected recurrence.

Case 1. A 37-year-old woman was treated with radiotherapy for stage II squamous cell carcinoma of the cervix in 1973. In 1975, she complained of low back pain radiating to the right leg. There was no clinical evidence of recurrent disease: The intravenous urogram revealed right-sided hydroureter and hydronephrosis. Pelvic angiogram revealed recurrent carcinoma in the right pelvic wall (Fig. 1). At laparotomy, exploration of the right pelvic wall confirmed the presence of recurrent disease. She was unsuccessfully treated with chemotherapy.

Case 2. A 45-year-old woman completed a course of radiotherapy for stage IB cervical carcinoma in May, 1975. She was seen in October 1975 because of low back pain. There was no clinical evidence of recurrence. An intravenous urogram revealed right hydroureter and hydronephrosis (Fig. 2A). The pelvic angiogram was normal (Fig. 2, B and C). A laparotomy and right pelvic wall lymph node dissection in January 1976 revealed no evidence of recurrent carcinoma. She has remained well with no evidence of disease.

\section{CONCLUSIONS}

1. Pelvic arteriography is a valuable method of assessing and identifying patients with suspected, persistent or recurrent cervical cancer, particularly those at the pelvic wall.

2. Tumor vessels and tumor stain are the most important angiographic diagnostic criteria. "Encasement" may be caused either by recurrent tumor or pelvic fibrosis.

\section{ACKNOWLEDGMENT}

This work was supported in part by grant ST01CA05120-14, the National Cancer Institute, Bethesda, MD, USA.

\section{REFERENCES}

1. Athanasoulis CA, Waltman AC, Barnes AB, Herbst AL: Angiographic control of pelvic bleeding from treated carcinoma of the cervix. Gynecol Oncol 4:144, 1976.

2. Bree RL, Goldstein HM, Wallace S: Transcatheter embolization of the internal iliac artery in the management of neoplasms of the pelvis. Surg Gynecol Obstet 143:597, 1976.

3. Conrady J, Elkin M, Romney SL, San Fillipo LJ: Pelvic angiography and lymphangiography in the evaluation of patients with carcinoma of the cervix. Surg Gynecol Obstet 122:983, 1966.

4. Cunningham JJ, Fuks Z, Castellano RA: Radiographic manifestations of carcinoma of the cervix and complications of its treatments. Radiol Clin North Am 11:(1)93, 1974.

5. Lang EK: Arteriography in gynecology. Radiol Clin North Am 5:133, 1967.

6. Lang EK: Arteriographic diagnosis of extension of carcinoma of the cervix into the vesico-vaginal septum. J La State Med Soc 120:333, 1968.

7. Lang EK, Greer JL: The value of pelvic arteriography for the staging of carcinoma of the cervix. Radiology 92:1027, 1969.

8. Llorens AS: Control of pain in pelvic cancer. Gynecol Oncol 4:133, 1976.

9. Meng CH, Elkin M: Gynecologic angiography. Semin Roentgenol 4:(3), July 1969.

10. Petty WM, Teaford AK, Park RT, Patow WE: Angiographic evaluation of early carcinoma of the cervix. Gynecol Oncol 1:211, 1973.

Address for reprints:

Mahmood Yoonessi

State University of New York at Buffalo

School of Medicine

Dept of Gynecology-Obstetrics

The Buffalo General Hospital

Buffalo, NY 14203

USA 